Diabetologe $2021 \cdot 17: 367-368$

https://doi.org/10.1007/s11428-021-00754-8

Angenommen: 10. März 2021

(c) Springer Medizin Verlag GmbH, ein Teil von Springer Nature 2021

\section{Matthias Laudes}

Lehrstuhl für Innere Medizin - Endokrinologie, Diabetologie und klinische Ernährungsmedizin, Klinik für Innere Medizin 1, Universitätsklinikum Schleswig-Holstein, Campus Kiel, Kiel, Deutschland

\title{
Mikrobiom und Mensch
}

Seit mehreren Jahrhunderten wird versucht, durch eine Beeinflussung von Darmbakterien günstige Effekte auf die menschliche Gesundheit zu entfalten. Einen regelrechten Schub erfuhr die medizinische Forschung diesbezüglich vor etwa 10-15 Jahren. Der Grund hierfür ist v. a. technischer Natur: In der Vergangenheit wurden Darmbakterien im Wesentlichen über Kultivierungstechniken charakterisiert. Da jedoch viele dieser Mikroorganismen Anaerobier sind, ließen sich damit nur maximal $10 \%$ der Populationen erfassen. Die Kultivierung wurde vor 10-15 Jahren durch moderne Hochdurchsatzsequenzierungstechniken ersetzt. Damit werden Bakterienpopulationen über das Genom charakterisiert, wobei die Summe aller Mikroorganismengene als Mikrobiom bezeichnet wird.

Bei der Hochdurchsatzsequenzierung kommen 2 unterschiedliche Ansätze zur Anwendung: Bei der 16s-rRNA-Sequenzierung (16s-rRNA: ribosomale Ribonukleinsäure mit der Größe 16S) werden nur Teile des bakteriellen Genoms analysiert (sog. variable Regionen), die zur Unterscheidung einzelner Bakterienarten ausreichend sind. Hingegen wird als Metagenom das Resultat der Sequenzierung des gesamten bakteriellen Genmaterials bezeichnet, was über den Nachweis von Genen für bestimmte Enzyme auch die Abschätzung mikrobieller Stoffwechselwege ermöglicht.

Anfangs wurden 2 verschiedene Möglichkeiten diskutiert, wie das Darmmikrobiom die menschliche Physiologie beeinflusst: Bei der „energy-harvest theory“ geht man davon aus, dass bestimmte Darmbakterien unverdauliche Nahrungsbestandteile (z. B. Ballaststoffe) vorverdauen, sodass zusätzliche Energie- substrate resorbiert werden können (z. B. kurzkettige Fettsäuren [,short chain fatty acids“; SCFA]). Hingegen wird bei der „inflammatory theory“ angenommen, dass bestimmte Darmbakterien immunologische Effekte in der Darmwand auslösen, die sich dann über den gesamten menschlichen Organismus ausbreiten, um über die sog. metabolische Entzündung z. B. Typ-2-Diabetes und Atherosklerose zu triggern. In jüngerer Vergangenheit wurde jedoch eine komplexere Vorstellung bezüglich der Interaktion von Bakterium und Mensch (sog. „host-microbe interaction“) entwickelt. Dabei geht man davon aus, dass ein Kometabolismus besteht, dass also der bakterielle und der menschliche Stoffwechsel eng miteinander verwoben sind.

》) Bakterieller und menschlicher Stoffwechsel sind wohl eng miteinander verwoben, zu einem Kometabolismus

Dies kann positive und negative Effekte bedingen: Bakterien können z. B. Trimethylaminoxid (TMAO) produzieren, welches als bakterieller Metabolit die Atherosklerose fördert und somit pathogene Eigenschaften im menschlichen Wirt entfaltet. Daneben können Bakterien - z. B. als Reaktion auf Metformin - den Metaboliten Agmatin synthetisieren, welchem aktuell positive Effekte auf die Insulinempfindlichkeit zugeschrieben werden.

Die Untersuchung des Darmmikrobioms wird zukünftig nicht nur in der Medizin eine große Bedeutung einnehmen, sondern auch in den Ernährungswissenschaften. In der Vergangenheit 
führten viele Ernährungsinterventionen zu keinen klaren Ergebnissen, was Effekte auf die menschliche Gesundheit und die Krankheitsentstehung betrifft. Heute wissen wir, dass jeder Mensch unterschiedlich auf Ernährungsinterventionen reagieren kann, in Abhängigkeit vom individuellen Mikrobiom. Trägt ein Proband A z. B. Darmbakterien, die aus einer bestimmten Ernährung vermehrt TMAO synthetisieren, und Proband B nicht, hat die gleiche Ernährungsintervention unterschiedliche Effekte auf die menschliche Physiologie. Dies bedeutet, dass Ernährungsstudien in Zukunft nicht nur den Zusammenhang Ernährung $\rightarrow$ Mensch adressieren sollten, sondern vielmehr den Zusammenhang Ernährung $\rightarrow$ Mikrobiom $\rightarrow$ Mensch, was aller Voraussicht nach künftig eindeutigere Ergebnisse liefern wird.

In dieser Ausgabe möchten wir Ihnen einen Überblick über die aktuellen Daten zur Rolle des Mikrobioms bei den Erkrankungen und Therapien, die uns Diabetologen in der täglichen Praxis begegnen, und einen Ausblick auf mögliche neue Therapieansätze unter Nutzung des Mikrobioms geben.

\section{Korrespondenzadresse}

Univ.-Prof. Dr. med.
Matthias Laudes
Lehrstuhl für Innere
Medizin - Endokrinologie,
Diabetologie und klinische
Ernährungsmedizin, Klinik
für Innere Medizin 1,
Universitätsklinikum
Schleswig-Holstein, Campus
Kiel
Düsternbrooker Weg 17,
24105 Kiel, Deutschland
matthias.laudes@uksh.de

Interessenkonflikt. M. Laudes hält zusammen mit anderen Wissenschaftlern und der Universität Kiel ein Patent zur Beeinflussung des Mikrobioms über eine topische intestinale Freisetzung von Niacin, PCT/EP2017/058733.

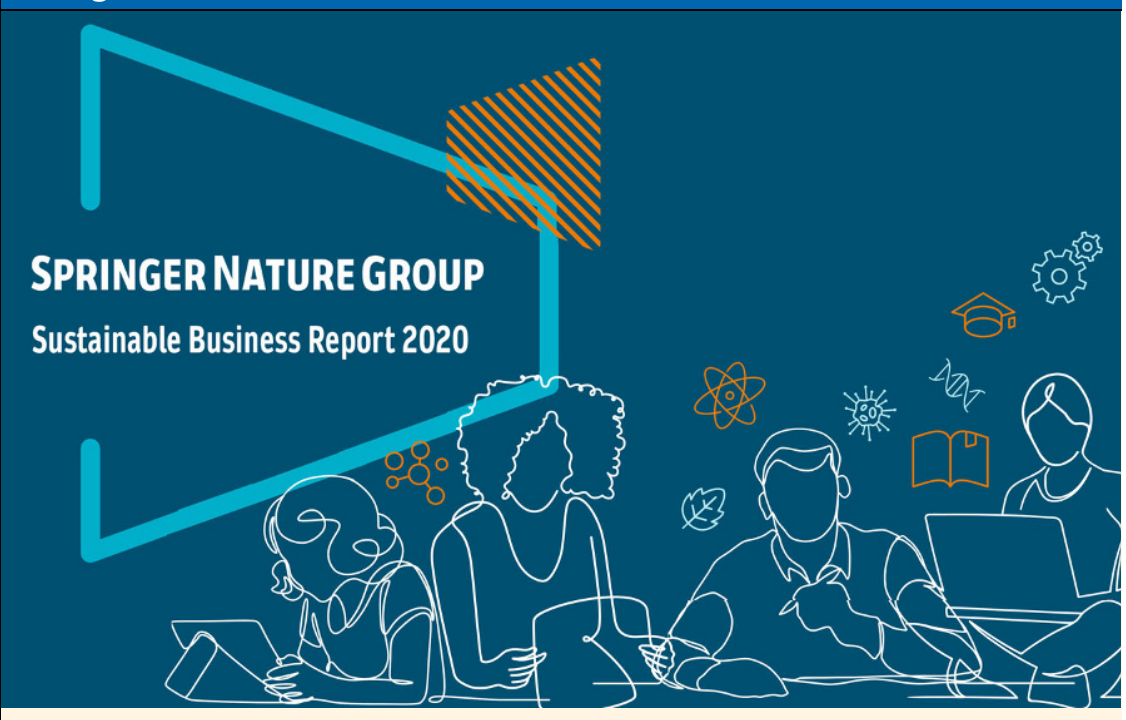

\section{Nachhaltigkeit bei Springer Nature}

Unser Sustainable Business Report 2020 zeigt, welche Fortschritte wir in den Bereichen Umwelt, Soziales und Unternehmensführung über die letzten Jahre erzielen konnten und berücksichtigt dabei auch externe Einflüsse wie die außergewöhnliche Situation im Jahr 2020 durch die COVID-19-

Pandemie. Als Unternehmen, das nachhaltig und verantwortungsvoll denkt und handelt wollen wir einen positiven Beitrag leisten. Verantwortung ist einer unserer Grundwerte und wir sind bestrebt, als nachhaltiges und ethisches Unternehmen zu handeln, das sich verpflichtet, die Auswirkungen seiner Tätigkeit auf die Umwelt zu berücksichtigen. Wir sind überzeugt, dass die von uns veröffentlichten Inhalte wichtige Gespräche und Diskussionen ermöglichen, die sich positiv auf Gesellschaft und Umwelt auswirken und wollen dieses Potential weiter ausbauen.

Im März 2020 haben wir uns dazu verpflichtet, klimaneutral zu werden. Bereits Ende 2020 haben wir dieses Ziel erreicht und sind - im Hinblick auf unsere Büroräumlichkeiten, Fahrzeugflotte und Geschäftsreisen - klimaneutral. Möglich wurde dies durch die erheblich gesteigerte Nutzung von Ökostrom und CO2-Ausgleichszahlungen. Als Verlag, der aktuellste Forschungsergebnisse zum Klimawandel veröffentlicht, wollen wir außerdem die Aufmerksamkeit für dieses Thema stärken - extern, aber auch intern bei rund 10.000 Beschäftigten, die weltweit bei Springer Nature arbeiten.
Durch den Einsatz neuer Technologien wurden 2020 mehr als drei Milliarden Artikel und Buchkapitel über unsere Plattformen heruntergeladen und damit 50\% mehr als 2019. Für uns ist Technologie der Schlüssel, um Wissen schneller, nachhaltiger und kostengünstiger für noch mehr Menschen in aller Welt verfügbar zu machen.

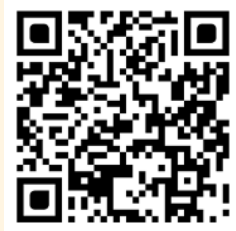

Mit dem QR-Code zur Kurzzusammenfassung des Nachhaltigkeitsreport.
Unsere Veröffentlichung im Jahr 2020:

- 3.000+ Zeitschriften

- 372.000+ Forschungsartikel

- 13.000+ Neue Bücher

- 100 Neue Lehrbuchreihen, die von mehr als 30 Millionen Studierenden genutzt werden 